\title{
Analog Sliding Mode Controller for Position Tracking of Piezoelectric Actuators
}

\author{
S. Yannier and A. Sabanovic \\ Sabanci University, Faculty of Engineering and Natural Sciences, \\ Mechatronics Engineering Program \\ Istanbul, Turkey
}

\begin{abstract}
In this work, analog application for the sliding mode control (SMC) of piezoelectric actuators is presented. DSP application of the algorithm suffers from ADC and DAC conversions and faces speed limitations. Moreover, piezoelectric actuators are known to have very large bandwidth close to the DSP operation frequency. Therefore, with the direct analog application, improvement of the performance is expected and high frequency operation will be achieved. First an appropriate SMC is designed to have continuous control output and then experimental results for position tracking using DSP and analog application are presented for comparison.
\end{abstract}

\section{INTRODUCTION}

Piezoelectric actuators, based on crystalline effects, do not suffer from "stick slip" effect and theoretically provide unlimited resolutions. Therefore, they are already used in many applications to provide sub-micrometer resolution; ultrasonic motors, sports materials like skis and bikes [1], aerospace [2], hard disk drives [3] etc... Other main application of these ceramics is the scanning tunneling microscope (STM) and atomic force microscope (AFM)[4].

Precision control of piezoelectric actuators is hardly acquired due to highly nonlinear input/output behavior dominated by hysteresis behavior between electrical voltage and strain [5-7]. Hysteresis yields a rateindependent lag and residual displacement near zero input, significantly reducing the precision of the actuators [8]. Another undesired characteristic of piezoelectric actuators is the "creep effect" [7].

Most of the piezoelectric actuator applications require high precision motion control where closed loop control is the only answer. Despite that fact many attempts to drive the piezoelectric actuator as open loop system with compensation of the nonlinearities are investigated [5-8].

In order to design a control scheme without precise dynamic modeling some fuzzy logic and neural network solutions are investigated. However, due to the limited performance, this research area did not find much popularity [9].

On the other hand, sliding-mode control (SMC) is one of the effective nonlinear robust control approaches. One of the most important aspects is the ability to force the system to stay on so-called "sliding mode" that exists in a manifold. SMC provides system invariance to uncertainties once the system is in the sliding mode [10, 11]. Sliding mode is originally designed as system motion for dynamic systems whose essential open-loop behavior can be sufficiently modeled with ordinary differential equations. The discontinuous control action is generally referred as variable structure control (VSC). The resulting feedback system is defined in continuous time domain and governed by differential equations with discontinuous right hand sides.

Abidi et al. used SMC in conjunction with the disturbance observer for both position and force tracking in piezoelectric actuators [11]. In their work, Abidi et al. showed that discrete time SMC can track nanometer size references with strain gage feedback in the presence of a disturbance observer. Proven that the controller has error on the order of the square of the sampling time, improvements on the speed of the controller are required. Although DSP based controllers are easier to build and run, their speed is limited. Analog circuits based on large bandwidth op-amp circuits on the other hand, promise much more speed.

The aim of this work is to design appropriate control to drive piezoelectric actuators on analog control circuit. By the way speed limit of the DSP is expected to be overcome and improvement of the tracking performance is expected.

\section{THE SLIDING MOde CONTROL}

In this paper, we will consider dynamical systems that can be represented as a class of nonlinear systems linear with respect of control as described by the following equation

$$
\dot{x}=f(x)+B(x) u+d
$$

where $x^{T} \in \mathfrak{R}^{n}$ is the state vector, $u \in \mathfrak{R}^{m}$ is the control vector, $f(x) \in \mathfrak{R}^{n}$ is an unknown, continuous and bounded nonlinear function, $B(x) \in \mathfrak{R}^{n x m}$ is a known input matrix whose elements are continuous and bounded and $\operatorname{rank}\left(\left.B(x)\right|_{\forall x}=m\right.$, with $d \in \Re^{n}$ being an unknown, bounded external disturbance. Both $f(x) \in \mathfrak{R}^{n}$ and $d \in \mathfrak{R}^{n}$ satisfy the matching conditions and all their components are bounded $\left\|f_{i}(x)\right\|_{\forall x} \leq M \quad$ and $\left\|d_{i}(t)\right\|_{\forall t} \leq N$. Fully actuated mechanical systems belong to the class of systems described by (1). Such systems can be interpreted as $\mathrm{m}$ interconnected sub-systems $\ddot{x}_{i}=h_{i}\left(x_{i}, \dot{x}_{i}\right)+b_{i}\left(x_{i}, t\right) \cdot u_{i}+g_{i}\left(x_{i}, x_{j}\right), h_{i}\left(x_{i}, \dot{x}_{i}\right)$ in general represents Coulomb friction term, $g_{i}\left(x_{i}, x_{j}\right)$ represents the interaction term and is regarded as a disturbance. 
The aim is to determine the control input $u=\left[u_{1}, \ldots, u_{m}\right]^{T}$ such that the system states $x_{1}(t), \ldots, x_{n}(t)$ track the desired trajectories $x_{d_{1}}(t), \ldots, x_{d_{n}}(t)$ while control error satisfies selected dynamical constraints.

\section{A. Controller Design}

The controller will be designed in the SMC framework by firstly selecting a suitable sliding manifold that will ensure desired systems dynamics and then selecting control such that the Lyapunov stability conditions are satisfied. Selecting the Lyapunov function candidate in terms of the sliding function is a natural way of guaranteeing the sliding mode existence on the selected manifold and thus having desired closed loop dynamics. Finally, the necessary control input should be selected that will fulfill the requirements of the Lyapunov stability criteria.

\section{1) Sliding Manifold}

For system (1) the natural selection of the sliding manifold is in the following form

$$
\sigma=G e_{t}=0
$$

where tracking error vector is defined as $e_{t}=\left[e_{1}, \ldots, e_{n}\right]^{T} \in \mathfrak{R}^{n}, e_{i}=x_{d_{i}}-x_{i}$ and the sliding surface satisfies $\sigma=\left[\sigma_{i}, \ldots, \sigma_{m}\right]^{T} \in \mathfrak{R}^{m}, G \in \mathfrak{R}^{m \times n}$.

2) Computing the Necessary Control Input A Lyapunov Function candidate can be selected as

$$
V=\frac{1}{2} \sigma^{T} \sigma
$$

where, $V \in \mathfrak{R}$. This function can also be stated as $V=(1 / 2)\|\sigma\|_{2}^{2}$, where $\|\bullet\|_{2}$ indicates Euclidian norm with $V(0)=0$. The time derivative of the candidate Lyapunov function $\dot{V}$ should be negative definite. In order to use this condition in selection of the control, we may require that $\dot{V}$ satisfies some preselected form. Equating the time derivative of this function to a negative definite function like in (4),

$$
\dot{V}=-\sigma^{T} D \sigma-\mu \frac{\sigma}{\sigma^{T} \sigma}
$$

where $D$ is a positive definite symmetric matrix, and $\mu>0$ thus Lyapunov conditions are satisfied. By substituting (3) into (4), the following requirement is found.

$$
\sigma^{T}\left(\dot{\sigma}+D \sigma+\mu \frac{\sigma}{\sigma^{T} \sigma}\right)=0
$$

Therefore, for $\sigma \neq 0$, the control law can be calculated by satisfying the following equation.

$$
\left(\dot{\sigma}+D \sigma+\mu \frac{\sigma}{\sigma^{T} \sigma}\right)=0
$$

and the sliding mode conditions are satisfied. The discontinuous term can be selected as small in order to avoid chattering. It had been proven $[12,13]$ that in the discrete time implementation the sliding mode is guarantied with continuous control action. We are targeting the computer controller systems for which controller will be implemented in discrete-time so in our application the discontinuous term $\mu \frac{\sigma}{\sigma^{T} \sigma}$ will be omitted and we will be determining the control action that satisfies conditions $(\dot{\sigma}+D \sigma)=0$ but all further analysis can be easily adopted for application of expression (6) if the term $D \sigma$ is replaced with $D \sigma+\mu \sigma / \sigma^{T} \sigma$.

For system (1) with sliding mode manifold (2) the control that satisfies $(\dot{\sigma}+D \sigma)=0$ can be determined as

$$
\begin{aligned}
u & =-(G B)^{-1}\left(G\left(f+d-\dot{x}_{d_{i}}\right)-D \sigma\right) \\
& =u_{e q}+(G B)^{-1} D \sigma
\end{aligned}
$$

where $x_{d}=\left\lfloor x_{d_{1}}, \ldots ., x_{d_{n}}\right\rfloor$ and $u_{e q}$ is so-called equivalent control obtained as a solution of the equation $\dot{\sigma}=G B\left(u_{e q}-u\right)=0$. By substituting (7) into (1) the equations of motion of system (1) in manifold (2) are obtained as $\sigma=G e_{t}=0$ and the approach to this solution is governed by equation (6). This is a result of the specific structure of the plant (1) in which states are selected as the derivatives of the measurable outputs and each sub-block is represented in the canonical form.

To implement this control input, information about the plant dynamics and external disturbances are needed, which is hard to achieve. Hence, this solution needs the information on the equivalent control thus may be applied for the plants when $u_{e q}$ is known or can be estimated with sufficient accuracy. In this paper we will be using a fact proven in $[14,15]$ that the solution of the differential equation

$$
\tau \dot{z}+z=\left(u-(G B)^{-1} \cdot \dot{\sigma}\right)
$$

with small enough filtering time constant $\tau$ is close to the equivalent control. In this paper we will be using this result in order to avoid direct calculation of the equivalent control from $u_{e q}=-(G B)^{-1} G\left(f+d-\dot{x}_{d}\right)$ but instead to use approximated result $u_{e q}=z$.

Equation (8) can be used for small enough filtering time $\tau$ together with the control presented in equation (7) to obtain the simplified controller equation;

$$
\begin{aligned}
u & =\frac{u_{e q}}{\tau \cdot s+1}+(G B)^{-1} D \sigma \\
& =\frac{u}{\tau \cdot s+1}+K \cdot\left(D \sigma+\frac{\dot{\sigma}}{\tau \cdot s+1}\right)
\end{aligned}
$$

where $K=(G B)^{-1}$ and $u_{e q}$ is replaced with $u_{e q}=\frac{u+K \cdot \dot{\sigma}}{\tau \cdot s+1}$ according to equation (8).

\section{B. The Model of PZT}

In this work, a piezo-stage that consists of a piezo-drive integrated with a sophisticated flexure structure for motion 
amplification is used. The flexure structure is wire-EDMcut and is designed to have zero stiction and friction.

In addition to the absence of internal friction, flexure stages exhibit high stiffness and high load capacity. Flexure stages are also insensitive to shock and vibration. However, since the piezo-drive exhibits non-linear hysteresis behavior, the overall system will also exhibit the same behavior.

The dynamics of the piezo-stage can be represented by the following second-order differential equation coupled with hysteresis in the presence of external forces

$$
m_{e f f} \ddot{x}+c_{e f f} \dot{x}+k_{e f f} x=T(u(t)-h(x, u))-F_{e x t}
$$

where $m_{\text {eff }}, c_{\text {eff }}$ and $k_{\text {eff }}$ denotes the effective mass, effective damping and effective stiffness of the stage respectively, $y$ is the displacement of the stage from its equilibrium state, $T$ denotes the electromechanical transformation ratio, $u$ is the input voltage and $h(x, u)$ denotes the non-linear hysteresis that has been found to be a function of $x$ and $u$, and $F_{e x t}$ is the external force acting on the stage $[6,16]$.

The model represented by (10) shows that from the mechanical motion the hysteresis may be perceived as a disturbance force that satisfies matching conditions. This means that the sliding mode based control should be able to reject the influence of the hysteresis nonlinearity on the mechanical motion. At the same time it is obvious that the lumped disturbance consisting of the external force acting on the system and the hysteresis can be estimated, thus allowing the application of the disturbance rejection method in the overall system design.

\section{ANALOG SOLUTION DESIGN AND EXPERIMENTS}

\section{A. Analog Solution Design}

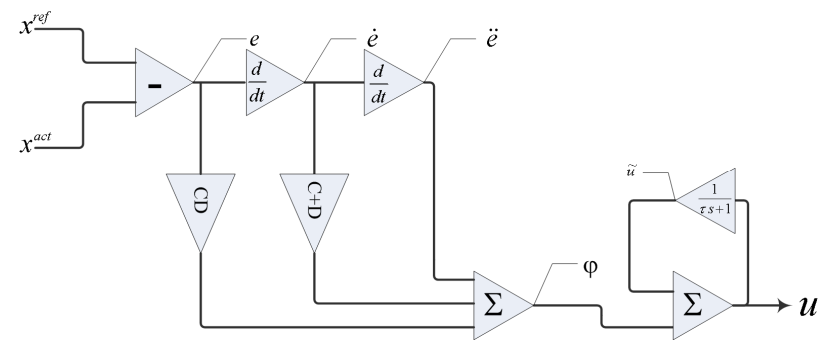

Figure 1 Draft analog circuit scheme to calculate the control.

For the control of the piezoelectric actuator with lumped model presented in section 2.2, using system states $x=\left[\begin{array}{ll}x & \dot{x}\end{array}\right]^{T} \in \mathfrak{R}^{2}$, the sliding manifold can be defined as;

$$
\sigma=G e_{t} \in \mathfrak{R}
$$

where tracking error vector is defined as $e_{t}=\left[\begin{array}{ll}e_{1} & e_{2}\end{array}\right]^{T} \in \mathfrak{R}^{2}, \quad e_{i}=x_{d_{i}}-x_{i}$ and $G \in\left[\begin{array}{ll}C & 1\end{array}\right]$ with $C \in \mathfrak{R}$.
For the analog application of the equation (9), using operational amplifier blocks, one must further simplify the equation before circuit design. Using equation (8),

$$
\begin{aligned}
& u=\tilde{u}+K \cdot(D C e+(D+C) \dot{e}+\ddot{e}) \\
& u=\tilde{u}+K \cdot \varphi
\end{aligned}
$$

is obtained by using $\tilde{u}=\frac{u}{\tau \cdot s+1}, e=e_{1}=x_{d}-x$ and $\dot{e}=e_{2}=\dot{x}_{d}-\dot{x}$.

Using this last equation, the design of the analog circuit starting from the calculation of the error is straight forward as depicted in Figure 1. However, there is one key issue about the signal magnitudes: the amplifier outputs (or signal strengths) cannot be numerically equal to the problem variables they represent, except in very special cases, since the outputs of the amplifiers are limited to the supply voltage, which is generally smaller than the problem variables. Therefore, represented signals may reach higher values than the supply voltage and saturate the outputs. Moreover, some gains used in the algorithm, namely the problem variables, may be too high or too small for a practical realization. Therefore, signal strengths cannot be numerically equal to the program variables, but merely proportional to them. They must be multiplied by appropriate coefficients, called "scale factors" to assure that the amplifier output or magnitude of the parameters is realistic.

TABLE I. ASSUMED MAXIMUM VALUES FOR SIGNALS AND PARAMETERS.

\begin{tabular}{|c|c||c|r|}
\hline Parameter & $\begin{array}{c}\text { Determined } \\
\text { Value }\end{array}$ & Signal & $\begin{array}{c}\text { Assumed } \\
\text { Max Value }\end{array}$ \\
\hline$C$ & $400 \leftrightarrow 500$ & $e$ & $0.05 \mathrm{~V}$ \\
$D$ & $40 \leftrightarrow 150$ & $\dot{e}$ & $1 \mathrm{~V}$ \\
$K$ & $O\left(10^{-6}\right)$ & $\ddot{e}$ & $100 \mathrm{~V}$ \\
& & $\varphi$ & $500 \mathrm{~V}$ \\
& & $u$ & $1 \mathrm{~V}$ \\
\hline
\end{tabular}

To determine the scaling factors, so called "analog scaling" technique is used as it was once used in analog computers. In this technique, each signal is normalized according to its estimated maximum value presented on the Table 1. Then signal equations are rewritten to find actual op-amp gains.

\section{1) Circuit Design}

Control presented on equation (12) can be applied using operational amplifier blocks. The designed circuit will be presented in three steps: preparation of the necessary variables $(e, \dot{e}$ and $\ddot{e})$, summation to find $\varphi$ and calculation of the control $u$.

The preparation of the variables $e, \dot{e}$ and $\ddot{e}$ for the calculation of the $\varphi$ consist of a difference amplifier that calculates the error $e$ from the desired and actual position data, $x_{d}$ and $x$ respectively, and two derivative blocks to calculate the first and second derivatives of the error. $\varphi$ is then calculated using a summing amplifier block with appropriate gains for each of the variables $e, \dot{e}$ and $\ddot{e}$. 


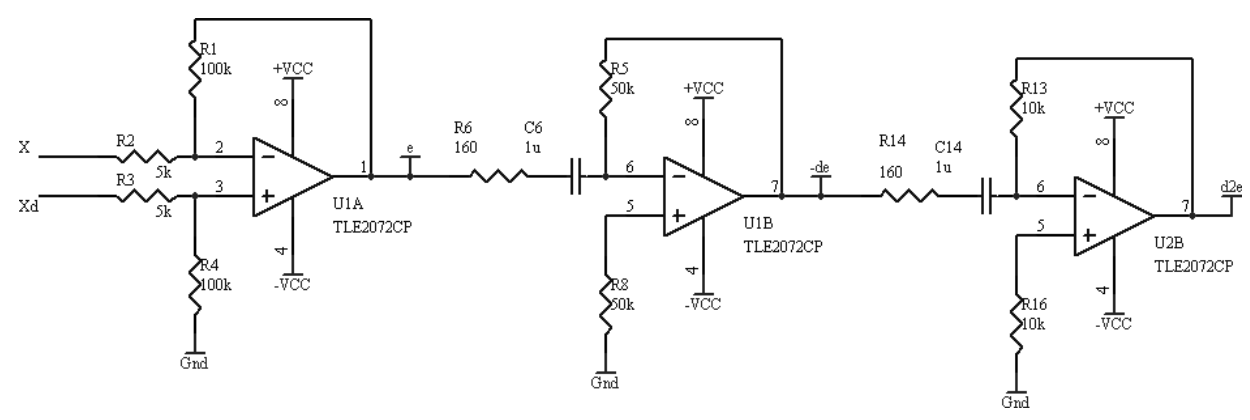

Figure 2 Preparation of the necessary variables ( $e, \dot{e}$ and $\ddot{e}$ ) from the desired and actual inputs; $x_{d}$ and $x$.

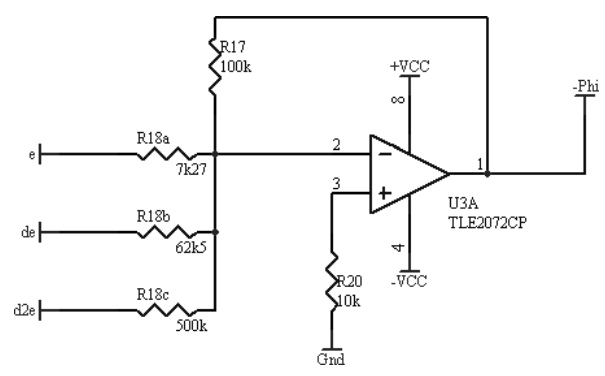

Figure 3 Calculation of $\varphi$ from previously found variables; $e, \dot{e}$ and $\ddot{e}$.

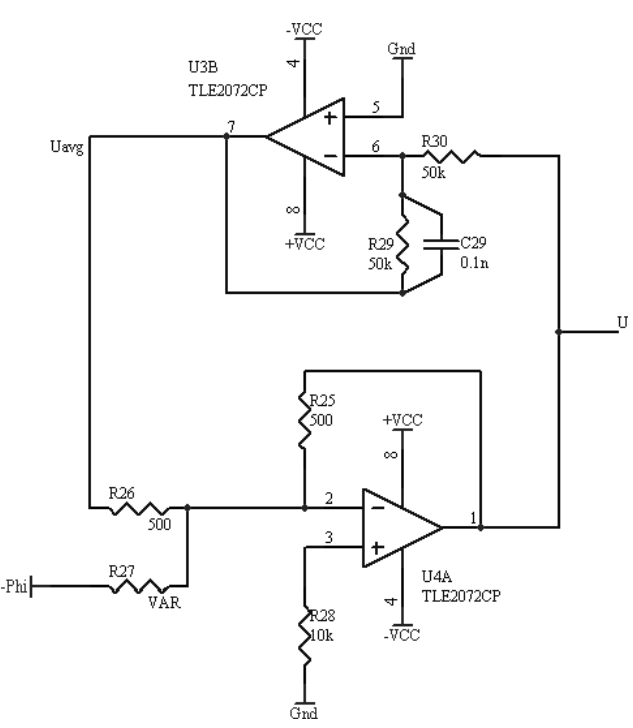

Figure 4 Calculation of $u=\tilde{u}+K \cdot \varphi$ where $\tilde{u}$ is found through the first order filtering of the control and $K$ is obtained by tuning the resistor $R_{27}$ since $K=R_{25} / R_{27}$.

At the last part, the calculation of the control $u$ is realized by $u=\tilde{u}+K \cdot \varphi$ as obtained in equation (12). $\tilde{u}$ is found through the first order filtering of the control where the filtering time constant has the order of $10^{-6}$.

The tuning of the parameters $C, D$ and $K$ is realized with variable resistors.

\section{PRELIMINARY EXPERIMENTS}

\section{A. Experimental Setup}

For experimental purposes, the setup shown in Figure 5 is constructed; voltage amplifier is the Piezomechanik SVR 150-3, PEA is the piezoelectric actuator with embedded strain gage for position measurement and the strain gage amplifier is the BA501 strain gage amplifier from Vishay's Measurement Group. Here SMC is the designed sliding mode control algorithm implemented in DSP (for DSP experiments) or is the analog circuit (for analog controller experiments).

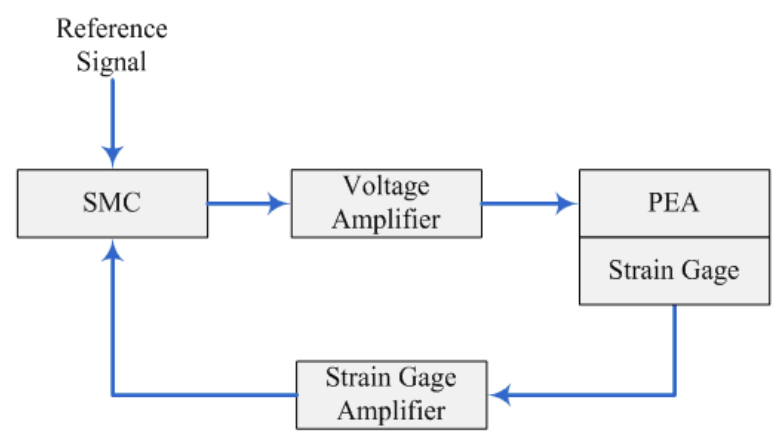

Figure 5 Piezoelectric actuator control setup.

Both in DSP and analog control experiments, the unknown structure of the high voltage amplifier assumed to be limited with a low pass filter.

The data is captured by Agilent Technologies 54622D digital oscilloscope. The reference and actual signals are given without offset to have better feeling on the tracking error. The third channel shown in figures is the difference between the signals, namely the error, as calculated by the oscilloscope. The scale of the signals is shown on the top left hand side part of the figures while the scale of the error is shown at the caption of each figure.

Naturally, voltages seen by the scope are the readings of the strain gage amplifier in terms of voltages. The performance can be better understood when those values are converted to metric correspondents: 17.96um (micrometers) corresponds to $1 \mathrm{~V}$ of the strain gage amplifier reading or in other words lum position deflection results $55.68 \mathrm{mV}$.

\section{B. Position Tracking Experiments Using DSP}

For comparison of the results DSP application of the control is realized on dSpace DS1102 platform which possesses TMS320C31 DSP chip running at $40 \mathrm{MHz}$ with 
50ns cycle time. The platform does have two 16-bit ADC (Input) $\pm 10 \mathrm{~V}$ and four 12-bit DAC (Outputs) $\pm 10 \mathrm{~V}$. The algorithm runs at $10 \mathrm{kHz}$. discretization is made based on Euler's method.

Position tracking of $1 \mathrm{~Hz}$ sinusoidal inputs is studied. First $4.5 \mathrm{um}$ and then $10.8 \mathrm{um}$ peak-to-peak inputs are tested. Results are shown on Figure 6 and on Figure 7 respectively. The errors for comparison are $110 \mathrm{~nm}$ and $200 \mathrm{~nm}$, corresponding to $2.5 \%$ and $1.9 \%$ respectively.

\section{Analog Circuit, Position Tracking Experiments}

Similar experiments are conducted for analog circuit realization of the SMC. The tracking of a $10.8 \mathrm{um}$ peak-topeak $1 \mathrm{~Hz}$ sinusoidal reference is successfully tracked with $360 \mathrm{~nm}$ peak-to-peak tracking error corresponding to $3.3 \%$ (Figure 8). Compare to $2.5 \%$ tracking error of the DSP implementation, this result is acceptable. The main source of the error is the noise that interferes starting from strain gage measurement to the calculation of the control. Derivative blocks on the other hand are known to amplify noise. Another source of the error is the offset introduced by the operational amplifiers. The offsets propagate through the circuit as a summation and cause a drift at the control output.

For better understanding of the performance, the step response and tracking of a triangular wave are also presented. Figure 9 shows that with careful adjustment of the circuit offset errors can be handled. Moreover with tuning of the control parameters $(C, D$ and $K$ ) tracking can be achieved for different plants.

Finally, Figure 10 is supplied to show the circuit performance on non-continuous reference tracking.

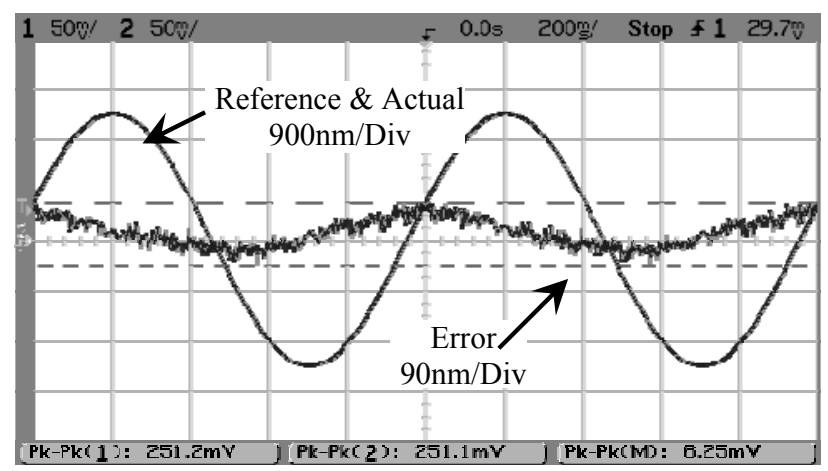

Figure 6 DSP tracking experiment for $4.5 \mathrm{um}$ pp $1 \mathrm{~Hz}$ sinusoidal reference.

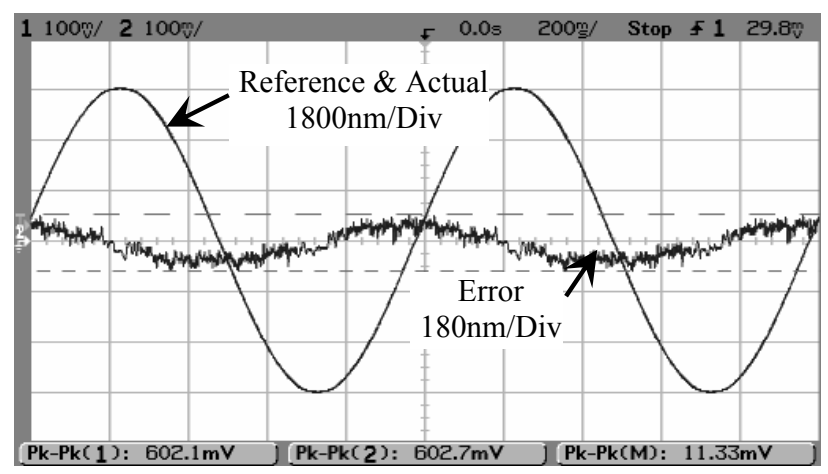

Figure 7 DSP tracking experiment for $10.8 \mathrm{um} \mathrm{pp} 1 \mathrm{~Hz}$ sinusoidal reference.

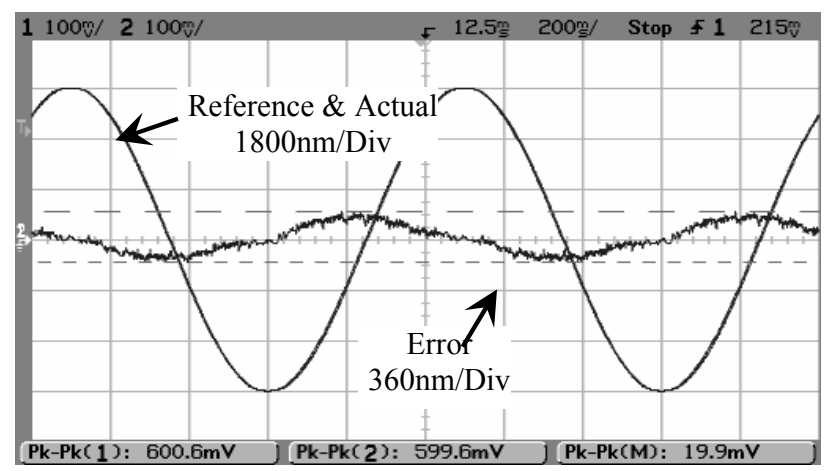

Figure 8 Analog circuit tracking experiment for $10.8 \mathrm{um}$ pp reference sinus of $1 \mathrm{~Hz}$.

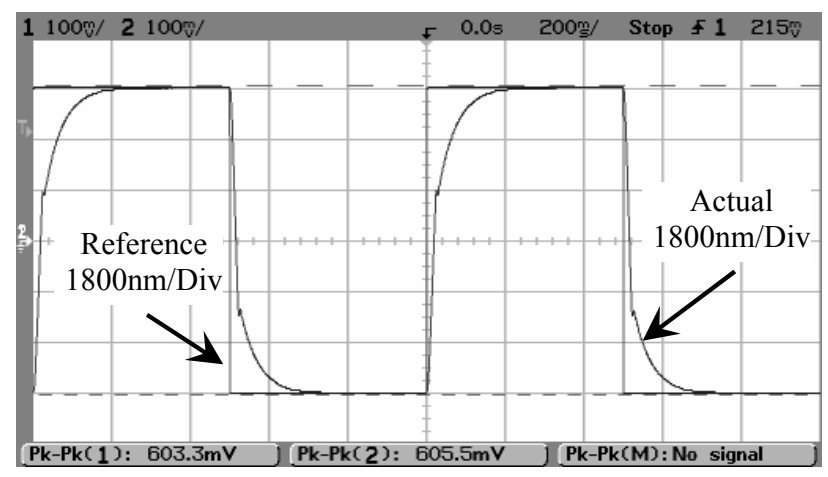

Figure 9 Analog circuit tracking experiment for $10.8 \mathrm{um}$ pp reference sinus of $1 \mathrm{~Hz}$.

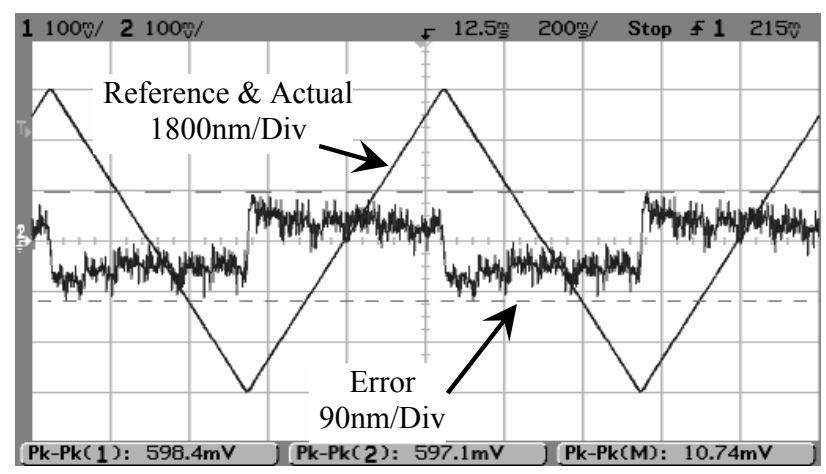

Figure 10 Analog circuit tracking experiment for $4.5 \mathrm{um}$ pp reference triangular wave.

\section{CONCLUSION}

In this paper we have formulated a SMC with continuous control output, based on [11, 13-15], applicable in analog electronics for the position tracking of the piezoelectric actuators. The use of such a controller in systems controlled by digital controllers, including DSP, PC, microchip and FPGA, will help users to save from heavy computational load. Moreover, due to the speed limitation in digital systems, mainly caused by the analog to digital conversion, the controller is expected to have better performance than the DSP application.

Experimental results proved that the analog production of the proposed SMC is possible and that the controller can track a reference signal with a good degree of accuracy. However, due to the fine-tuning difficulties 
together with noise interference the tracking error is almost equal to DSP implementation.

\section{REFERENCES}

[1] S. Ashley, "Digital dampers", in Mechanical Engineering, vol. 120: American Society of Mechanical Engineers, 1998, pp. 130.

[2] "Shape shifting aircraft", in Design Engineering: Centaur Communications, 2001, pp. 5.

[3] B. M. Chen, T. H. Lee, C.-C. Hang, Y. Guo, and S. Weerasooriya, "An Hinf almost disturbance decoupling robust controller design for a piezoelectric bimorph actuator with hysteresis", Control Systems Technology, IEEE Transactions on, vol. 7, pp. 160-174, 1999.

[4] H. Richter, E. A. Misawa, D. A. Lucca, and H. Lu, "Modeling nonlinear behavior in a piezoelectric actuator", Precision Engineering, vol. 25, pp. 128-137, 2001.

[5] H. J. M. T. S. Adriaens, W. L. De Koning, and R. Banning, "Modeling piezoelectric actuators", Mechatronics, IEEE/ASME Transactions on, vol. 5, pp. 331-341, 2000.

[6] M. Goldfarb and N. Celanovic, "Modeling piezoelectric stack actuators for control of micromanipulation", Control Systems Magazine, IEEE, vol. 17, pp. 69-79, 1997.

[7] H. Jung, H. Jung, J. Y. Shim, J. Y. Shim, D. Gweon, and D. Gweon, "New Open-loop Actuating Method of Piezoelectric Actuators for Removing Hysteresis and Creep", Review of Scientific Instruments, vol. 71, 2000.

[8] J.-J. Tzen, S.-L. Jeng, and W.-H. Chieng, "Modeling of piezoelectric actuator for compensation and controller design", Precision Engineering, vol. 27, pp. 70-86, 2003.
[9] R.-J. Wai and J.-D. Lee, "Intelligent motion control for linear piezoelectric ceramic motor drive", Systems, Man and Cybernetics, Part B, IEEE Transactions on, vol. 34, pp. 21002111, 2004.

[10] K. D. Young, V. I. Utkin, and U. Ozguner, "A control engineer's guide to sliding mode control", Control Systems Technology, IEEE Transactions on, vol. 7, pp. 328-342, 1999.

[11] K. Abidi, A. Sabanovic, and S. Yesilyurt, "Sliding mode control based disturbance compensation and external force estimation for a piezoelectric actuator", presented at Advanced Motion Control, 2004. AMC '04. The 8th IEEE International Workshop on, 2004.

[12] W.-C. Su, S. V. Drakunov, and U. Ozguner, "An $\mathrm{O}(\mathrm{T}<\sup >2</$ sup $>)$ boundary layer in sliding mode for sampled-data systems", Automatic Control, IEEE Transactions on, vol. 45, pp. 482-485, 2000.

[13] S. Drakunov and V. Utkin, "A semigroup approach to discretetime sliding modes", presented at American Control Conference, 1995. Proceedings of the, 1995.

[14] V. Utkin, "Variable structure systems with sliding modes", Automatic Control, IEEE Transactions on, vol. 22, pp. 212222, 1977.

[15] V. Utkin, J. Guldner, and J. Shi, Sliding Mode Control in Electro-mechanical Systems. London; Philadelphia, PA: Taylor \& Francis, 1999.

[16] M. Goldfarb and N. Celanovic, "Behavioral implications of piezoelectric stack actuators for control of micromanipulation", presented at Robotics and Automation, 1996. Proceedings., 1996 IEEE International Conference on, 1996. 